Service social

\title{
La cyberintimidation chez les jeunes : mieux comprendre pour mieux intervenir à l'école
}

\section{Jacques Quintin, Emmanuelle Jasmin et Elena Théodoropoulou}

Volume 62, numéro 1, 2016

Ensemble contre l'intimidation : une responsabilité partagée

URI : https://id.erudit.org/iderudit/1036332ar

DOI : https://doi.org/10.7202/1036332ar

Aller au sommaire du numéro

Éditeur(s)

École de service social de l’Université Laval

ISSN

1708-1734 (numérique)

Découvrir la revue

Citer cet article

Quintin, J., Jasmin, E. \& Théodoropoulou, E. (2016). La cyberintimidation chez les jeunes : mieux comprendre pour mieux intervenir à l'école. Service social, 62(1), 1-23. https://doi.org/10.7202/1036332ar
Résumé de l'article

Les nouveaux médias sociaux posent la question de l'identité et soulèvent de nouveaux enjeux éthiques. L'enjeu du bien-être des jeunes commande de penser autrement ces médias sociaux numériques en introduisant la réflexion éthique. À l'aide d'une recension des écrits, nos objectifs consistent à comprendre mieux la cyberintimidation en vue de proposer des stratégies éducatives. Le phénomène de la cyberintimidation est similaire à celui de l'intimidation. En revanche, les aspects public et anonyme associés à la cyberintimidation pourraient être vécus plus difficilement par les jeunes. De futures recherches devraient explorer davantage la dimension du sens que revêt la cyberintimidation pour les jeunes. 


\title{
La cyberintimidation chez les jeunes : mieux comprendre pour mieux intervenir à l'école
}

\author{
QUINTIN, Jacques, Ph.D. (philosophie) \\ JASMIN, Emmanuelle, Ph.D. (ergothérapie) \\ THÉODOROPOULOU, Elena, PH.D. (philosophie)
}

\section{RÉSUMÉ}

Les nouveaux médias sociaux posent la question de l'identité et soulèvent de nouveaux enjeux éthiques. L'enjeu du bien-être des jeunes commande de penser autrement ces médias sociaux numériques en introduisant la réflexion éthique. À l'aide d'une recension des écrits, nos objectifs consistent à comprendre mieux la cyberintimidation en vue de proposer des stratégies éducatives. Le phénomène de la cyberintimidation est similaire à celui de l'intimidation. En revanche, les aspects public et anonyme associés à la cyberintimidation pourraient être vécus plus difficilement par les jeunes. De futures recherches devraient explorer davantage la dimension du sens que revêt la cyberintimidation pour les jeunes.

Mots clés : intimidation, cyberintimidation, identité, bien-être, éthique, école.

\begin{abstract}
The new social media are bringing up the question of identity and raise new ethical issues. The youths' well-being requires different ways of thinking about these digital social media, namely through the introduction of ethical reflection. Our objectives, using a literature review, consist to better understand cyberbullying with a view to suggesting related educational strategies. The phenomenon of cyberbullying is similar to that of bullying. However, the public and anonymous aspects of cyberbullying may make it an even more difficult experience for young people. Future studies should further explore the dimension of the meaning of cyberbullying for young people. Keywords : bullying, cyberbullying, identity, well-being, ethics, school.
\end{abstract}




\section{Contexte social de la cyberintimidation}

Les médias sociaux sont des outils culturellement puissants (Turkle, 1995). S'ils permettent l'invisibilité et l'anonymat, ils rendent les actes d'intimidation et de cyberintimidation plus visibles. Ils posent ainsi un défi à nos cultures traditionnelles et à nos manières de penser l'identité (Turkle, 1997 ; Turkle, 2004).

C'est dans ce cadre qu'émerge le problème complexe de la cyberintimidation qui, justement, contrairement aux formes de la violence physique, est plus difficile à identifier par la famille et l'école chez les jeunes qui en sont victimes. Balançant entre une philosophie de la technologie et une étude sociologique, l'interprétation des événements, qui se développe dans l'espace des médias sociaux, doit tenir compte des particularités de cet espace. Ainsi, la cyberscience constatera que le web forme un système technosocial voué aux transformations posant des questions éthiques inusitées (Berners-Lee et al, 2006). Pour toutes ces raisons, l'étude du phénomène de cyberintimidation semble dépasser nos capacités d'observation, ce qui demeure pour la recherche une tâche fort compliquée. Dans cet espace particulier, comprendre d'une manière interdisciplinaire les causes ou mécanismes fondamentaux qui mènent à des actions d'agression et de violence devient nécessaire.

Il est important de souligner, par ailleurs, que les usagers des médias sociaux sont des citoyens qui partagent l'expérience de la citoyenneté active, du moins en partie, notamment en prenant position sur des enjeux d'actualité. Or, l'expérience numérique ne saurait qu'être, à la fois, une expérience personnelle et politique/éducative qui tend vers une nouvelle liaison entre l'individuel et le collectif (Puelles et Pereira, 2009). Benkler (2006) remarque que le nouvel environnement numérique développe des rapports symbiotiques de renforcement ou de dépendance mutuelle, rapports qui, selon le cas, sont positifs (le développement des collaborations) ou négatifs (le parasitisme, le pillage, etc.) ou même néfastes (cybercriminalité). En ce sens, l'écosystème numérique est une structure auto-organisée qui se caractérise par le savoir commun, les technologies ouvertes, les nouvelles communautés où les citoyens peuvent explorer, découvrir, dénoncer et produire de nouveaux modes de production de savoir pour le bien commun, ce qui crée un nouvel environnement pour la vie humaine (Benkler, 2006 ; Pereira, 2015). Il est significatif que le phénomène de cyberintimidation, tout en exploitant les possibilités ouvertes de la technoculture, rompt avec la dimension communautaire en y insérant le traumatisme de la violence qui ne peut être expliqué que par une interprétation interdisciplinaire combinant systématiquement tant les facteurs psychosociaux - au niveau individuel et au niveau 
collectif - qu'une herméneutique philosophique et politique de la technologie soutenant les médias sociaux. C'est à ce niveau que tant les recherches empiriques que les études théoriques, qui examinent ces questions particulières, sont encore peu nombreuses. La complexité et la singularité de la question de cyberintimidation expliquent d'une part cette rareté, mais d'autre part témoignent de la nécessité des approches systématiques, systémiques et détaillées.

L'étude moderne de l'intimidation remonte aux années 1970 avec Olweus en Suède (McGuinness, 2007). II existe maintenant plusieurs études sur les problèmes engendrés par l'intimidation, et cela, dans plusieurs contextes nationaux (Schuster, 1996). Depuis le début des années 2000, la société dans laquelle nous vivons s'est grandement modifiée en raison de l'utilisation massive des médias sociaux numériques, ce qui a engendré un nouveau phénomène, soit la cyberintimidation. C'est dans ce contexte qu'au Québec, une loi a été mise en vigueur depuis juin 2012, qui oblige les écoles à adopter et à mettre en place un plan de lutte contre l'intimidation et la violence à l'école, incluant la cyberintimidation.

Il existe plusieurs définitions de la cyberintimidation. Généralement la cyber-intimidation se caractérise par l'utilisation des médias sociaux (internet, téléphone portable, messagerie texte, réseaux sociaux comme Facebook et Twitter, etc.) pour exercer sur un tiers différentes agressions et abus de pouvoir, dont de l'intimidation (Li, 2006 ; Smith et al. 2008 ; Cassidy, Jackson et Brown. 2009 ; Hinduja et Patchin, 2009 ; Le Deuff et al., 2010) qui porte atteinte à la réputation et qui induit un sentiment d'humiliation, de peur et un sentiment de désespoir (Strom et Strom, 2005). Cela peut se faire en propageant des messages textes blessants, des fausses rumeurs, des photos compromettantes. Les messages textes envoyés sur les téléphones portables semblent être le médium le plus utilisé pour la cyberintimidation (Cross et al., 2009, cité dans Li, 2010). Nous comprenons l'intimidation comme «tout comportement, parole, acte ou geste délibéré ou non à caractère répétitif, exprimé directement ou indirectement, y compris dans le cyberespace, dans un contexte caractérisé par l'inégalité des rapports de force entre les personnes concernées, ayant pour effet d'engendrer des sentiments de détresse et de léser, blesser, opprimer ou ostraciser » (Gouvernement du Québec, 2012).

Loin d'être strictement des outils de communication très utiles, ces nouvelles technologies sont à risque de compromettre le bien-être des adolescents en raison d'une mauvaise utilisation. L'enjeu du bien-être des adolescents commande de penser autrement ces médias sociaux numériques en introduisant la réflexion éthique. 
De plus, ces nouveaux médias mettent à mal la frontière entre la sphère privée et la sphère publique. Il est d'usage pour les jeunes de se regrouper dans les espaces publics non médiatisés tels que les centres commerciaux, les squares et autres lieux publics. Les adolescents y rencontrent leurs amis et se lient à d'autres personnes. «Les réseaux sociaux numériques ne sont qu'une forme particulière d'espace public » (Boyd, 2010, p. 48). II n'en demeure pas moins qu'ils induisent des changements majeurs. Contrairement à ce qui se passe dans les espaces publics traditionnels, ce qui est déclaré s'inscrit durablement. II existe une persistance des messages. Malgré le caractère anonyme apparent, il est possible d'effectuer un traçage des traces laissées sur les médias sociaux. De plus, l'information numérique est reproductible à l'infini. Enfin, les observateurs sont invisibles. « S'il est courant de se trouver face à des inconnus dans l'espace public de tous les jours, nos yeux permettent de savoir qui écoute ou entend ce que nous disons. Dans un espace public numérique, non seulement les observateurs sont invisibles, mais la persistance, le traçage et la reproductibilité ouvrent la porte à des observateurs qui n'étaient même pas présents au moment où le message a été émis » (Boyd, 2010, p.49). II n'y a plus de cloisons franches entre la sphère privée et la sphère publique. « Les conservations se diffusent et les contextes disparaissent »(Boyd, 2010,p. 49). On assiste à une perte de contrôle. C'est dans ce contexte que se déploient de nouvelles formes d'intimidation comme la cyberintimidation.

À l'aide d'une recension des écrits, notre objectif consiste à comprendre mieux le phénomène de la cyberintimidation en vue de proposer une stratégie éducative pour réduire ce phénomène.

Cette recension des écrits repose sur trois formes de recherche, reconnues dans les écrits en méthodologie de la recherche : 1) la recherche informatisée ; 2) la recherche manuelle dans des ouvrages de référence ; 2) l'examen des références bibliographiques des articles recensés (Fortin, et Gagnon, 2010; Mertens, 2010). Nous avons consulté diverses banques de données pour réaliser la recherche informatisée, dont : Pubmed, PsycINFO, CINAHL, CAIRN. Les motsclés suivants ont été utilisés en français et en anglais, même si la littérature sur le sujet est principalement anglophone: cyber-tindimidation/cyberbulling, éthique/ethics, santé mentale/mental health, identité/identity, bien-être/well-being et ennui/boring.

\section{L'intimidation comparée à la cyberintimidation}

Si l'intimidation se présente comme un phénomène universel (Eslea et al., 2004), la cyberintimidation (i.e. l'intimidation à l'aide des médias sociaux) est une nouvelle forme 
d'intimidation qui est observée dans tous les pays où se sont déroulées des études sur la cyberintimidation ( $\mathrm{Li}, 2010)$. Les études montrent qu'entre $9 \%$ et $50 \%$ des élèves sont victimes de cyberintimidation (Beran et Li, 2005 ; Ybarra et al., 2006 ; David-Ferdon et Hertz, 2007, 2009 ; Wolak, Mitchell et Finkelhor, 2007 ; Li, 2007; Smith et al., 2008 ; Tokunaga, 2010 ; Patchin et Hinduja, 2012 ; Cappadocia, Craig et Peppler, 2013).

Les recherches concluent que la cyberintimidation est moins fréquente que l'intimidation traditionnelle (Williams et Guerra, 2007 ; Smith et al., 2008 ; Wang, lannoti et Nasel, 2009). Par contre, il existe un recoupement entre la cyberintimidation et les formes d'intimidation traditionnelle. C'est pour cette raison que nous exposons conjointement les formes d'intimidation et de cyberintimidation. Des chercheurs pensent que la cyberintimidation est un dérivé de l'intimidation traditionnelle (Dehue, Bolman et Völlink, 2008) tandis que d'autres ont suggéré que la cyberintimidation est un genre d'intimidation distinct (Slonje et Smith, 2008). En fait, il y a peu d'études qui permettent de comparer la cyberintimidation avec l'intimidation traditionnelle (Perren et al., 2010). Par exemple, selon Schneider (2012) et d'autres chercheurs (Beran et Li, 2005), les intimidateurs, puisqu'ils peuvent ne pas être dans une relation de face à face avec les victimes, peuvent ne pas reconnaître la violence de leur comportement. II est peu probable qu'ils expérimentent des sentiments de regret, de sympathie et de compassion. Contrairement aux pratiques d'intimidation plus traditionnelle qui se déroulaient dans un contexte plus limité, voire privé, où les événements sont connus seulement par un groupe restreint, les messages textes et les images peuvent être communiqués à un large public dans une période de temps très courte, de sorte que les intimidateurs peuvent éviter toutes formes de responsabilité pour leur geste et croire qu'ils ne se feront pas prendre en défaut et être punis (Cooper, 2012 ; Kowalski et Limber, 2007 ; Junoven et Gross, 2008 ; Mishna, Saini et Solomon, 2009).

L'étude de Sticca et Perren (2013) montre, à partir de scénarios fictifs, que les jeunes perçoivent l'intimidation publique et l'intimidation anonyme pires que l'intimidation en privé et l'intimidation non anonyme. Les scénarios de cyberintimidation étaient perçus en général comme pires que les scénarios d'intimidation traditionnelle. Toutefois, le média serait secondaire. Ce sont les aspects public et anonyme, plus fréquemment associés à la cyberintimidation, qui dérangent avant tout.

Nous savons que les victimes de la cyberintimidation sont aussi victimes d'intimidation plus traditionnelle (Ybarra, Diener-West et Leaf, 2007 ; Juvonen et Gross, 2008 ; Cross et al. 2009 ; Dooley, Pyzalski et Cross, 2009 ; Gradinger, Strohmeier et Spiel, 2009 ; Twyman et al., 2010 ; Li, 
2010) et présentent les mêmes symptômes. Selon l'étude de Cappadocia, Craig et Peppler (2013), les jeunes victimes d'intimidation traditionnelle sont plus à risque de subir et de participer à la cyberintimidation. L'expérience antérieure d'intimidation traditionnelle pourrait donc être un facteur de risque de la cyberintimidation. Cependant, nous ignorons si les conséquences de la cyberintimidation sont aussi négatives que celles issues de l'intimidation traditionnelle (Perren et al., 2010) ou pires. Li (2010) pense qu'il est possible que les dommages induits par la cyberintimidation soient plus grands que ceux générés par l'intimidation traditionnelle dans la mesure où les victimes ne peuvent pas se cacher.

Une caractéristique commune est observée autant chez les intimidateurs que chez les victimes d'intimidation, soit le manque d'habiletés sociales (Rincon-Robichaud, 2003). L'année de transition au secondaire et la présence de dépression seraient des facteurs de risque associés à la cybervictimisation (Cappadocia, Craig et Peppler, 2013). Un degré élevé de comportements antisociaux, une faible influence prosociale des pairs et une consommation élevée d'alcool seraient associés à un risque élevé de participation à la cyberintimidation (Cappadocia, Craig et Peppler, 2013).

Selon une méta-analyse, les victimes de cyberintimidation seraient portées à devenir ellesmêmes des cyberintimidateurs (Kolwalski et al., 2014). La participation à l'intimidation traditionnelle serait modérément corrélée à la participation à la cyberintimidation. Deux prédicteurs modérément reliés à l'engagement dans la cyberintimidation seraient les croyances normatives concernant l'agression et le désengagement moral. La participation à la cyberintimidation serait aussi modérément liée à la consommation d'alcool et de drogue. Les cyberintimidateurs seraient plus susceptibles de rapporter une diminution de la satisfaction de vie, de l'estime de soi et de la réussite scolaire ainsi qu'un degré plus élevé de solitude. Avoir été victime d'intimidation traditionnelle serait le plus grand prédicteur d'être également victime de cyberintimidation. Les jeunes victimes de cyberintimidation auraient aussi davantage tendance à participer à l'intimidation traditionnelle (Kowalski et al., 2014).

\section{La différence des genres}

L'intimidation et la cyberintimidation sont liées à l'identité. De son côté, l'identité est reliée à la question du genre. Mais, comme l'indique Tokunaga (2010), la différence des genres n'est pas claire. Certaines études, et elles sont peu nombreuses (Seals et Young, 2003), montrent que les garçons sont victimes de cyberintimidation en plus grand nombre que les filles (Nansel et al., 
2001 ; Carlyle et Steinma, 2007 ; Varjas, Heinrich et Meyers, 2009) tandis que d'autres études montrent le contraire (Kowalski et Limber, 2007 ; Wang, lannoti et Nasel, 2009). D'autres études n'y voient aucune différence (Mynard et Joseph, 2000). Il est difficile de cerner la différence entre les genres en raison de la difficulté de cerner les modalités de l'intimidation (Boulton et Underwood, 1992 ; Borg, 1999).

Selon l'étude de Cappadocia, Craig et Peppler (2013), le taux de participation à la cyberintimidation serait équivalent chez les filles et les garçons. Toutefois, les filles seraient davantage victimes de cyberintimidation, comparativement aux garçons. En revanche, d'autres études arrivent à des résultats contradictoires (Kowalk et al., 2014). D'après quelques études, les filles seraient davantage cyberintimidées par les courriels alors que les garçons le seraient plus par les messages textes (Kowalski et al., 2014).

L'intimidation peut être motivée par une attitude ou un comportement discriminatoire basé notamment sur l'orientation sexuelle, la présence d'une déficience ou d'une incapacité, le groupe ethnique, la classe sociale ou l'apparence physique. À ce jour, la plupart des études sur la cyberintimidation ont porté sur des enfants sans caractéristiques particulières (Kowalski et al., 2014).

\section{Les conséquences d'être victime de cyberintimidation}

Peu d'études se sont penchées sur les conséquences de la cyberintimidation. Par contre, il existe plusieurs études qui montrent les effets de l'intimidation. II existe une corrélation entre l'intimidation et des indicateurs négatifs tels qu'une moins bonne réussite scolaire, une plus faible satisfaction de fréquenter l'école et un engagement plus bas envers l'école (Dake, Price et Telljohann, 2003 ; Spriggs et al., 2007).

La relation entre les pairs est d'une grande importance pour le développement de l'identité, la santé et le bien-être des enfants (Ladd, 2005). Dans ce contexte, il est bien établi qu'être victime d'intimidation entraîne des effets négatifs à court et à long terme. Ces effets négatifs sont la solitude, l'insatisfaction sociale et le retrait social (Kochenderfer et Ladd, 1996), des difficultés émotionnelles et comportementales (Perren et al., 2006 ; Kowalski et Limber, 2007 ; Raskauskas et Stoltz, 2007 ; Hinduja et Patchin, 2008, 2009), les symptômes dépressifs (Hodges et Perry, 1999 ; Reader Goodman, Stormshak et Dishion, 2001; Hanish et Guerra, 2002 ; Gazelle et Ladd, 2003 ; Ybarra, 2004, Ybarra et al., 2006). Être victime d'intimidation par ses pairs induit une faible estime de soi (Alsaker et Olweus, 2002), des problèmes de santé (Nishina, Juvonen et Witkow, 
2005), des idées suicidaires et des troubles d'adaptation scolaire (Storch et al., 2003). L'expérience de la cyberintimidation induit aussi des abus de substances (Goebert et al., 2011).

Les médias sociaux font état de plusieurs cas de suicide en raison de la cyberintimidation (Kennedy, 2010). Plusieurs études se sont intéressées sur la relation entre la cyberintimidation et le suicide (idée suicide et acte suicidaire) (Brunstein Klomek et al., 2007 ; Brunstein Klomek et al., 2008 ; Kim et Leventhal, 2008 ; Brunstein Klomek, Sourander et Gould, 2010 ; Hinduja et Patchin, 2010). II ressort que la cyberintimidation, comparée à l'intimidation plus traditionnelle, est davantage liée au suicide (Gini et Espelage, 2014). Malgré les nombreuses études sur le rapport entre la cyberintimidation et le suicide, les enseignants et les directions d'école ont une représentation inadéquate de la situation (Trachtenbroit, 2011). Comme le note Cooper, Clements et Holt (2012), l'intimidation comme la cyberintimidation ne peuvent plus être considérées comme un simple rite de passage.

\section{Les conséquences d'adopter des comportements de cyberintimidation}

Li (2007) démontre dans une étude que $15 \%$ des adolescents posent des actes de cyberintimidation. Les jeunes personnes qui posent des gestes d'intimidation sont aussi aux prises avec des conséquences négatives similaires aux victimes d'intimidation (Mason, 2008). En plus des gestes d'intimidation, Moffitt (1993) montre que ces jeunes adoptent aussi d'autres comportements antisociaux. On observe également chez eux davantage de problèmes physiques et psychologiques que chez leurs pairs (Kumpalainen, Räsänen et Henttoten, 1999) ainsi qu'un risque élevé de dépression et d'idéation suicidaire (Kaltiala-Heino et al., 1999). Enfin, ceux qui posent des gestes d'intimidation et qui sont aussi en même temps victimes d'intimidation montrent un niveau élevé de symptômes de détresse (Menesini, Modena et Tani, 2009). Sullivan (2000) montre qu'il existe une corrélation entre l'adoption de comportements d'intimidation et l'acquisition de relations instables. Comparés à la population générale, les hommes intimidateurs maltraitent leur conjointe plus fréquemment, conduisent leur voiture de manière plus erratique, perdent leur emploi régulièrement et posent un plus grand nombre de délits. Les femmes intimidatrices sont plus disposées à maltraiter leur progéniture. Il y a un taux d'alcoolisme, de troubles de personnalité plus élevé chez les hommes et les femmes qui adoptent des comportements d'intimidation. Ceux-ci ont davantage besoin d'utiliser les services de santé mentale. 


\section{L'enjeu de l'identité}

Nous vivons dans une culture numérique. Cela n'est pas aussi simple « que l'usage le laisse parfois paraître » (Le Deuff et al., 2010, p. 42). Si l'utilisation des médias sociaux pour les adultes répond à un besoin d'information, les médias sociaux constituent pour les jeunes « des extensions de soi, des excroissances utilisées pour satisfaire non pas un besoin d'information mais plutôt un besoin d'affirmation » de soi (Le Deuff et al., 2010, p. 42). Il y va de son identité personnelle et de son identité sociale (en lien avec le sentiment d'appartenance).

Internet fait désormais partie des agents de socialisation des jeunes (Denis et al., 2013). Ainsi, il contribue à la construction de l'identité sociale des jeunes. Les médias sociaux, par l'élaboration de profils, peuvent mener à une extériorisation de soi, où la personnalité virtuelle prend parfois le dessus sur la personnalité réelle. Cela peut mener à la création d'identité et dans l'anonymat, au dévoilement de l'intimité. Cependant, la majorité utiliserait leur vraie identité : $92 \%$ des enfants et adolescents utiliseraient leur vraie identité et diffuseraient un grand nombre d'information personnelle. La diffusion d'information personnelle entraîne cependant des risques, comme la cyberintimidation (Denis et al., 2013).

L'adolescence est un temps important de la vie où le développement de l'identité prend son essor. Les médias sociaux numériques sont perçus par les adolescents comme faisant partie de leur identité (Baumeister et Leary, 1995 ; Turkle, 1995 ; Katz, 2003 ; Calvert, 2002 ; Livingstone, 2008 ; Li, 2010). L'adolescence peut être marquée par un sentiment d'insécurité, éprouvé par le jeune qui cherche à définir sa place ou son rôle dans la société (Denis et al., 2013). Le jeune prend une distance vis-à-vis de ses parents, afin de se définir en tant que personne indépendante. Son groupe d'amis devient alors son milieu de référence. L'influence du groupe d'amis peut être positive ou négative (Denis et al., 2013). Selon l'étude de Hinduja et Patchin (2013), les comportements de cyberintimidation chez les adolescents sont à la fois influencés par leurs pairs et les adultes signifiants dans leur vie. Les jeunes ont tendance à participer davantage à l'intimidation et la cyberintimidation, s'ils pensent que leurs pairs se conduisent de manière similaire. En revanche, ils seraient moins portés à y participer, s'ils croient qu'ils seront sanctionnés par les adultes dans leur vie.

Durant la période de l'adolescence, en lien avec la quête d'identité, l'amitié prend de nouvelles proportions plus importantes (Tassoni, 2007). Les adolescents cherchent les situations et les comportements qui les aident à s'apprécier positivement et cherchent à éviter ceux qui les 
rendent mal à l'aise concernant leur identité (Patchin et Hinduja, 2010). Comme le mentionne Ling et al. (2003), le téléphone portable permet de se lier, d'amitié peu importe l'endroit et le temps. D'ailleurs, ceux qui sont victimes de cyberintimidation en informent seulement leurs amis (Li, 2010). Les amis sont probablement les meilleures personnes pour aider les victimes à gérer la détresse générée par la cyberintimidation (Hodges et Perry, 1999 ; Bukowski, 2001 ; Erdley et al., 2001; Jeffrey, Miller et Linn, 2001).

Ensuite, il existe une relation de réciprocité entre les expériences émotionnelles et la conscience de soi (Silvia et Eddington, 2012). Les états émotionnels affectent l'expérience de soi de la même façon que la conscience de soi affecte la nature des expériences émotionnelles. C'est pourquoi les formes d'intimidation, qui génèrent des états de détresse, induisent une réévaluation de l'identité de la personne de manière négative (Ogilvie, 1987). Également, les gestes d'intimidation induisent chez les élèves des croyances négatives à propos de leur identité, du monde dans lequel ils vivent et de leur avenir. Ces croyances négatives peuvent causer la dépression (Beck, 1967 ; Ingram, 1984).

La plupart des recherches sont centrées sur l'identification de la prévalence de la cyberintimidation chez les adolescents. Très peu se sont intéressées sur le vécu de la cyberintimidation en lien avec l'identité. La faible estime de soi sert parfois à expliquer le phénomène de la cyberintimidation (Egan et Perry, 1998 ; Salmivalli et al., 1999 ; Glover et al., 2000 ; Wild et al., 2004 ; Yang et al., 2010 ; Frisen, Jonsson et Persson, 2007 ; Beaty et Alexeyev, 2008 ; Jankauskiene et al., 2008), même s'il est difficile de départager la cause de l'effet. Est-ce la faible estime de soi qui cause l'intimidation ou est-ce le fait d'être une victime d'intimidation qui entraîne une faible estime de soi (Egan et Perry, 1998) ? De plus, il existe peu d'études sur la relation entre l'estime de soi et la cyberintimidation (Patchin et Hinjuda, 2010). Selon d'autres chercheurs (Rigby et Slee, 1991 ; Salmivalli et al., 1999 ; Baumeister et Vohs, 2003), il en va autrement, car il existerait a contrario une forte relation entre une estime de soi surévaluée et l'adoption de comportement d'intimidation. Les études montrent que les individus avec une estime de soi irréaliste vont attaquer ceux et celles qui oseraient défier leur image de soi. Par conséquent, les interventions concentrées davantage sur la maîtrise de soi que sur l'estime de soi connaissent plus de succès. 


\section{Interventions et différence générationnelle}

Il est fréquemment recommandé aux jeunes de signaler à leurs parents le fait qu'ils sont victimes de cyberintimidation. Pourtant moins de $35 \%$ signalent les incidents de cyberintimidation aux adultes (Li, 2007). Cottle (2001) relève que les jeunes se montrent plutôt réticents à dévoiler cet aspect de leur vie à leurs parents, car ils craignent que leurs parents leur confisquent leur ordinateur, leur accès à internet ou leur téléphone portable. Ce qui aurait comme conséquence d'isoler l'adolescent, de créer un mal-être chez lui et peut-être de nuire à la formation de son identité. Cela suscite un questionnement éthique à savoir si les adultes doivent limiter temporairement l'accès à ces médias sociaux (Kowalski, Limber et Agatston, 2008).

Il existe une différence générationnelle qui explique en partie pourquoi les conseils provenant des adultes sont simplistes et ont peu d'influence chez les adolescents. La plupart des adultes pensent que les ordinateurs et les médias sociaux sont des outils pratiques qui peuvent être utilisés pour rechercher, envoyer et recevoir de l'information. II en va autrement pour les adolescents. Ils considèrent ces médias comme un aspect essentiel de leur vie sociale, autant pour la création que le maintien de liens d'amitié (Oksman et Rautiainen, 2002). Le clavardage est l'activité électronique numéro un chez les adolescents (Roberts et Foehr, 2003).

Ce qui est bien vu par les adultes, par exemple créer son profil sous la forme d'un curriculum vitae, ne l'est pas nécessairement pour les adolescents (Boyd, 2010). Certains adultes vont recommander aux adolescents d'utiliser de fausses données comme le nom, l'âge, l'adresse. Cette attitude pose un questionnement éthique dans la mesure où elle encourage le mensonge comme moyen pour résoudre des conflits. Une autre stratégie utilisée par les adultes consiste à diaboliser les médias sociaux comme la source de bien des maux. Par conséquent, ils recommanderont d'éviter leur utilisation (Gunthert, 2010).

\section{Le rôle de l'école et ses enjeux éthiques en lien avec l'éducation citoyenne}

La Loi oblige les écoles québécoises à adopter et à mettre en place un plan de lutte contre l'intimidation et la violence. Au fil des années sont apparues plusieurs stratégies pour contrer le phénomène de l'intimidation et de la cyberintimidation avec des taux de succès mitigés, quoique significatifs. Plusieurs études qui ont évalué la portée des stratégies mises en place constatent une amélioration qui se situe entre 20 et $30 \%$. La plupart des stratégies mises en place l'ont été 
par des adultes pour des adolescents comme si tous les adolescents pensaient ou devaient penser comme des adultes.

Selon une recension systématique des écrits, les programmes scolaires d'intervention contre l'intimidation devraient s'inspirer du programme norvégien d'Olweus (Ttofi et Farrington, 2009). Les principaux éléments associés à une diminution de l'intimidation à l'école incluent la formation aux parents, la supervision lors des jeux à l'extérieur, les méthodes disciplinaires, les conférences à l'école, l'information aux parents, les règlements en classe et la gestion de la classe. Selon l'étude d'Elledge et al. (2013), l'attitude individuelle et collective des élèves de même que la compétence de l'enseignant à intervenir lors d'incidents d'intimidation en classe réduiraient les risques de cyberintimidation. II existe des outils de sensibilisation pour les jeunes pour prévenir l'intimidation, par exemple la trousse : «On s'élève : Outil de sensibilisation au potentiel éducatif des personnes handicapées » de l'Office des personnes handicapées du Québec (OPHQ). Néanmoins, à notre connaissance, peu d'outils existent pour la prévention de la cyberintimidation.

Si nous désirons prévenir la cyberintimidation et améliorer le climat scolaire, des efforts holistiques doivent être instaurés (Hinduja et Patchin, 2009). Les effets bénéfiques d'un sain climat scolaire sont bien documentés (Haynes, Emmon et Ben-Avie, 1997 ; Payne, Gottfredson et Gottfredson, 2003 ; Stover, 2005). Dans ce contexte, il devient pertinent que les élèves reçoivent une formation à l'utilisation des médias sociaux « qui dépasse le seul bon usage et le suivi de bonnes pratiques » (Le Deuff et al., 2010).

Il est attendu que l'école doit fournir un environnement sécuritaire pour les élèves qui y vivent. Une formation à l'utilisation des médias sociaux numériques implique aussi une démarche éthique, notamment dans sa relation à l'autre. En cela, il s'agit de la penser dans une dimension citoyenne et pas seulement sur le plan des compétences (Jenkins et al., 2008). C'est pourquoi, même si les adultes recommandent souvent aux adolescents de falsifier leur identité pour se protéger, il devient nécessaire de s'interroger sur l'utilisation du mensonge comme moyen de résoudre ou de prévenir certaines difficultés liées à l'utilisation des médias sociaux.

Le besoin d'affirmation de soi et le besoin de se construire une identité impliquent une relation à l'autre qui ne va pas de soi. C'est quelque chose qui s'apprend et qui passe par le respect des autres. Il s'agit donc de développer le sens du prendre soin de l'autre. « Prendre soin, ici, signifie aussi faire attention, et d'abord porter et prendre attention à soi-même, et par la même occasion aux siens, et aux amis des siens, et donc de proche en proche à tous : aux autres quels qu'ils 
soient, et au monde que l'on partage avec eux en sorte que la formation d'une telle attention constitue une conscience d'universalité fondée sur (et profanée par) une conscience de singularité » (Stiegler, 2008, p. 139).

Il est manifeste que les médias sociaux numériques offrent moins de confidentialité que les moyens traditionnels de communication. Une raison de plus de former sur les enjeux de la confidentialité et du respect de la vie privée. Par exemple, le transfert d'images ne devrait pas se faire sans une autorisation des personnes concernées.

Nous devons nous demander si les enseignants (l'établissement scolaire) ont l'obligation (morale) d'éduquer les élèves quant à la manière d'utiliser les médias sociaux numériques. Les élèves ont besoin de savoir que toutes les formes d'intimidation, dont la cyberintimidation, ne sont pas acceptables (Willard, 2007a, 2007b). Doit-on pour autant instaurer des mesures disciplinaires ? Si oui, quelles en seront les conséquences ? Une étude a fait ressortir qu'un climat disciplinaire dans lequel les élèves internalisent les normes et les valeurs de l'école, et se conforment à celles-ci, réduit la fréquence de l'intimidation parmi les adolescents (Ma, 2001). Prendre conscience très tôt dans le parcours scolaire (au niveau primaire), des conséquences d'une mauvaise utilisation des médias sociaux numériques permettrait des comportements plus responsables.

\section{Conclusion}

Les médias sociaux, bien implantés dans nos sociétés, impliquent une réflexion sur leur utilisation, particulièrement en lien avec le phénomène de la cyberintimidation. La recension des écrits révèle plusieurs similitudes entre l'intimidation et la cyberintimidation, notamment les conséquences négatives sur l'identité, l'estime de soi et le bien-être. En revanche, les aspects public et anonyme associés à la cyberintimidation pourraient être vécus plus difficilement par les jeunes.

La plupart des études se sont intéressées à la prévalence de la cyberintimidation. Très peu ont posé leur attention sur l'expérience des adolescents et au sens qu'ils accordent à cette expérience de la cyberintimidation. Pour cette raison, les futures recherches devraient explorer davantage la dimension du sens que revêt la cyberintimidation pour les jeunes. De plus, nous avons très peu d'information sur les stratégies adoptées par les jeunes pour se protéger, pour prévenir et combattre l'intimidation. II serait donc pertinent d'explorer les moyens qu'ils recommanderaient pour prévenir et combattre la cyberintimidation. 
Une autre piste à étudier serait l'ennui vécu par les jeunes. Selon la recherche de Sharp et al. (2006), plus les jeunes vieillissent, moins ils sont motivés et engagés dans des activités de loisir. L'ennui a également été associé à des comportements agressifs (Dahlen et al., 2006), de même qu'à la consommation excessive d'alcool (Caldwell et Smith, 1995). Étant donné l'absence d'études à ce sujet en lien avec la cyberintimidation, ce facteur reste à explorer.

\section{QUINTIN, Jacques, Ph.D. (philosophie)}

Département de psychiatrie

Faculté de médecine et des sciences de la santé Université de Sherbrooke (Canada)

JASMIN, Emmanuelle, Ph.D. (ergothérapie) Département d'ergothérapie, École de réadaptation Faculté de médecine et des sciences de la santé Université de Sherbrooke (Canada)

THÉODOROPOULOU, Elena, PH.D. (philosophie) Dép. des sciences de l'éducation préscolaire et d'ingénierie éducative Faculté des Sciences Humaines Université d'Égée (Grèce)

\section{RÉFÉRENCES}

Alsaker, F.D., et D. Olweus (2002). « Stability and change in global self-esteem and self-related affect » in T.M. Brinthaupt et R.P. Lipka (dir.), Understanding Early Adolescent Self and Identity: Applications and Interventions, Albany (NY), State of New York University Press, p. 193-223.

Baumeister, R.F., et K.D. Vohs (2003). « Self-regulation and the executive function of the self », in M.R. Leary et J.P. Tangney (dir.), Handbook of Self and Identity, New York, Guilford, p. 197217.

Baumeister, R.F., et M.R. Leary (1995). «The need to belong: desire for interpersonal attachements as a fundamental human motivation », Psychological Bulletin, vol. 117, $\mathrm{n}^{\circ} 3$, p. 497-529.

Beaty, L.A., et E.B. Alexeyev (2008). « The problem of school bullies: what the research tells us », Adolescence, vol. 43, $\mathrm{n}^{\circ}$ 169, p. 1-11.

Beck, A.T. (1967). Depression: Clinical, Experimental, and Theoritical Aspects, New York, Harper \& Row.

Belsey, B., «Cyberbullying: An Emergent Threat to the "Always On" Generation », <www.bullying.org>. Consulté le 15 janvier 2014. 
Benkler, Y. (2006). The Wealth of Networks: How Social Production Transforms Markets and Freedom, New Haven (CT), Yale University Press.

Beran, T., et Q. Li (2005). « Cyber-harassment: A New Method for an Old Behavior », Journal of Education Computing Research, vol. 32, n³, p. 265-277.

Berners-Lee, T., W. Hall, J.A. Hendler, K. O'Hara, N. Shadbold, et D.J. Weitzner (2006). « A Framework for Web Science », Foundations and Trends in Web Science, vol. 1, n 1, p. 1-130.

Borg, M.G. (1999). "The extent and nature of bullying among primary and secondary schoolchildren », Educational Research, vol. 41, n² 2, p. 137-153.

Boulton, M.J., K. Underwood (1992). « Bully/victim problems among middle school children », British Journal of Educational Psychology, vol. 62, p. 73-87.

Boyd, D. (2010). «Réseaux sociaux : privés, publics, ou quoi ? », Documentaliste. Science de l'Information, vol. 47, $\mathrm{n}^{\circ} 1$, p. 48-49.

Brunstein Klomek, A., F. Marrocco, M. Kleinman, I.S. Schonfeld, et M.S. Gould (2007). « Bullying, depression, and suicidality in adolescents », Journal of the American Academy of Child and Adolescent Psychiatry, vol. 46, nº 1, p. 40-49.

Brunstein Klomek, A., A. Sourander, et M. Gould (2010). « The association of suicide and bullying in childhood to young adulthood: a review of cross-sectional and longitudinal research findings », Canadian Journal of Psychiatry, vol. 55, n 5, p. 282-288.

Brunstein Klomek, A., F. Marrocco, M. Kleinman, I.S. Schonfeld et M.S. Gould (2008). « Peer victimization, depression, and suicidality in adolescents », Suicide Life Threat Behavior, vol. 38, n² 2, p. 166-180.

Bukowski, W.M. (2001). « Friendship and the Worlds of Childhood » New Directions for Child and Adolescent Development, vol. 91, p. 93-106.

Caldwell, L.L., et E.A. Smith (1995). « Health Behaviors of Leisure Alienated Youth », Society and Leisure, vol. 18, n 1, p. 143-156.

Calvert, S.L. (2002). «Identity construction on the Internet », in S.L. Calvert, A.M. Jordan et R.R. Cocking (dir.) Children in the Digital Age: Influences of Electronic Media on Development, Westport (CT), Praeger, p. 57-70.

Cappadocia, M.C., W.M. Craig et D. Peppler (2013). « Cyberbullying: Prevalence, Stability and Risk Factors during Adolescence », Canadian Journal of School Psychology, vol. 28, $\mathrm{n}^{\circ} 2$, p. 171-192.

Carlyle, K.E., et K.J. Steinma (2007). «Demographic differences in the prevalence, cooccurrence, and correlates of adolescent bullying at school », Journal of School Health, vol. $77, n^{\circ} 9$, p. 623-629.

Cassidy, W., M. Jackson, K.N. Brown (2009). « Sticks and Stones Can Break my Bones, But How Can Pixels Hurt Me? Students' Experiences with Cyber-Bullying », School Psychology International, vol. 30, $\mathrm{n}^{\circ} 4$, p. 383-402. 
Cottle, T.J. (2001). Mind Fields: Adolescent Consciousness in a Culture of Distraction, New York, Peter Lang.

Cooper, G.D., P.T. Clements et K.E. Holt (2012). « Examining childhood bullying and adolescent suicide: Implications for school nurses », The Journal of School Nursing, vol. 28, n 4, p. 275283.

Cross, D., T. Shaw, M. Epstein, H. Monks, L. Lester et L. Thomas (2009). Australian Covert Bullying Prevalence Study, Perth, Child Health Promotion Research Centre, Edith Cowan University.

Dahlen, E.R., R.C. Martin, K. Ragan et M.M. Kuhlman (2006). « Boredom proneness in anger and aggression: effects of impulsiveness and sensation seeking », Personality and Individual Differences, vol. 37, nº 8, p. 1615-1627.

Dake, J.A., J.H. Price et S.K. Telljohann. (2003). « The nature and extent of bullying at school », Journal of School Health, vol. 73, $\mathrm{n}^{\circ}$ 5, p. 173-180.

David-Ferdon, C., et M. Feldman Hertz (2009). Electronic Media and Youth Violence: A CDC Issue Brief for Researchers, Atlanta (GA), Centers for Diseases Control and Prevention.

David-Ferdon, C. et M. Feldman Hertz (2007). « Electronic media, violence, and adolescents: An emerging public health problem », Journal of Adolescent Health, vol. 41, n 6, p. S1-S5.

Dehue, F., C. Bolman et T. Völlink (2008). «Cyberbullying: Youngsters' Experiences and Parental Perception », Cyberpsychology \& Behavior, vol. 11, $\mathrm{n}^{\circ} 2,217-223$.

Denis, C., G. Millette, J. Quérin et I. Vekeman-Julien (2013). Individu et société (5e éd.), Montréal, Chenelière Éducation.

Dooley, J.J, J. Pyzalski et D. Cross (2009). « Cyberbullying Versus Face-to-Face Bullying. A Theoretical and Conceptual Review », Zeitschrift für Psychologie/Journal of Psychology, vol. 217, no 4, p. 182-188.

Egan, S.K. et D.G. Perry (1998). «Does low self-regard invite victimization? », Developmental Pscyhology, vol. 34, n 2, p. 299-309.

Elledge, L.C., A. Williford, A.J. Boulton, K.J. DePaolis, T.D. Little et C. Salmivalli (2013). «Individual and Contextual Predictors of Cyberbullying: The Influence of Children's Provictim Attitudes and Teachers' Ability to Intervene », Journal of Youth Adolescence, vol. 42, no 5, p. 698-710.

Erdley, C.E., D.W. Nangle, J.E. Newman et E.M. Carpenter (2001). « Children’s Friendship Experiences and Psychological Adjustment: Theory and Resarch », New Directions for Child and Adolescent Development, vol. 91, n 1, p. 5-24.

Eslea M. de, E. Menesini, Y. Morita, M. O'Moore, J.A. Mora-Merchan, B. Pereira et P.K. Smith (2004). «Friendship and loneliness among bullies and victims: Data from seven countries », Aggressive Behavior, vol. 30, $\mathrm{n}^{\circ}$ 1, p. 71-83. 
Fortin, M.-F. et J. Gagnon (2010). Fondements et étapes du processus de recherche : Méthodes quantitatives et qualitatives ( $2^{\mathrm{e}}$ éd.), Montréal, Chenelière Éducation.

Frisen, A., A.K. Jonsson et C. Persson (2007). « Adolescents' Perception of Bullying: Who is the Victim? Who is the Bully? What Can Be Done to Stop Bullying? », Adolescence, vol. 42, $\mathrm{n}^{\circ}$ 168, p. 749-761.

Gazelle, H., et G.W. Ladd (2003). «Anxious Solitude and Peer Exclusion: A Diathesis-Stress Model of Internalizing Trajectories in Childhood », Child development, vol. 74, $\mathrm{n}^{\circ} 1$, p. 257278.

Gini, G., et D.L. Espelage (2014). « Peer victimization, cyberbullying, and suicide risk in children and adolescents, Journal of the American Medical Association, vol. 312, n 5, p. 545-546.

Glover, D., G. Gough, M. Johnson et N. Cartwright (2000). « Bullying in 25 secondary schools: incidence, impact and intervention », Educational Research, vol. 42, n² 2, p. 141-156.

Goebert, D., I. Else, C. Matsu, J. Chung-Do et J.Y. Chang (2011). « The impact of cyberbullying on substance use and mental health in a multiethnic sample », Maternal and Child Health Journal, vol. $15, \mathrm{n}^{\circ} 8$, p. 1282-1286.

Gouvernement du Québec (2012). Projet de loi $n^{\circ} 56$ : Loi visant à combattre l'intimidation et la violence à l'école.

Gradinger, P., D. Strohmeier et C. Spiel (2009). «Traditional Bullying and Cyberbullying: Identification of Risk Groups for Adjustment Problems », Zeitschrift für Psychologie/Journal of Psychology, vol. 217, $\mathrm{n}^{\circ}$ 4, p. 205-213.

Gunthert, A. (2010). «Le portrait numérique », Documentaliste. Sciences de l'Information, vol. $47, n^{\circ} 1$, p. 50-51.

Hanish, L.D. et N.G. Guerra (2002). « A longitudinal analysis of patterns of adjustment following peer victimization », Development and Pschopathology, vol. 14, n 1, p. 69-89.

Haynes, N.M., C. Emmons et M. Ben-Avie (1997). «School Climate as a Factor in Student Adjustment and Achievement », Journal of Educational and Psychological Consultation, vol. 8, $n^{\circ} 3$, p. 321-329.

Hinduja, S., et J.W. Patchin (2010). « Bullying, cyberbullying, and suicide », Archives of Suicide Research, vol. 14, n 3, p. 206-221.

Hinduja, S., et J.W. Patchin (2009). Bullying Beyond the Schoolyard: Preventing and Responding to Cyberbullying, Thousand Oaks (CA), Sage Publications.

Hinduja, S., et J.W. Patchin (2008). « Cyberbullying: An Exploratory Analysis of Factors Related to Offending and Victimization », Deviant Behavior, vol. 29, n² 2, p. 129-156.

Hinduja, S., et J.W. Patchin (2013). « Social Influences on Cyberbullying Behaviors Among Middle and High School Students », Journal of Youth and Adolescence, vol. 42, n ${ }^{\circ}$ 5, p. 711722. 
Hodges, E.V.E., et D.G. Perry (1999). «Personal and interpersonal antecedents and consequences of victimization by peers », Journal of Personality and Social Psychology, vol. $76, n^{\circ} 4$, p. 677-685.

Ingram, R.E. (1984). «Toward an information-processing analysis of depression », Cognitive Therapy and Research, vol. 8, n 5, p. 443-477.

Jankauskiene, R., K. Kardelis, S. Sukys et L. Kardeliene (2008). « Associations between school bullying and psychosocial factors », Social Behavior and Personality, vol. 36, n 2, p. 145-162.

Jeffrey, L., P. Miller et M. Linn (2002). « Middle school bullying as a context for the development of passive observers to the victimization of others », in R.A. Geffner, M. Loring et C. Young (dir.), Bullying Behavior: Current Issues, Research, and Interventions, p. 143-156, New York (NY), Routledge.

Jenkins, H., K. Clinton, R. Purushotma, A.J. Robison et M. Weigel (2008). Confronting the Challenges of Participatory Culture: Media Education for the 21st Century, MacArthur foundation,<https://www.macfound.org/media/article_pdfs/JENKINS_WHITE_PAPER.PD>.

Juvonen, J. et E.F. Gross (2008). « Extending the school grounds?-Bullying experiences in cyberspace », Journal of School Health, vol. 78, n 9, p. 496-505.

Kaltiala-Heino, R., M. Rimpelä, M. Marttunen, A. Rimpelä et P. Rantanen (1999). « Bullying, depression, and suicidal ideation in Finnish adolescents: school survey », British Medical Journal, vol. 319, nº 7216, p. 348-351.

Katz, J.E. (dir.) (2003). Machines that Become Us: The Social Context of Personal Communication Technology, New Brunswick (NJ), Transaction Publishers.

Kennedy, H. (2010). « Phoebe Prince, South Hadley High School's "new girl" driven to suicide by teenage cyber bullies », New York Daily News, 29 mars 2010.

Kim, Y.S., et B. Leventhal (2008). « Bullying and suicide. A review », International Journal of Adolescent Medicine and Health, vol. 20, $\mathrm{n}^{\circ}$ 2, p. 133-154.

Kochenderfer, B.J., et G.W. Ladd (1996). « Peer Victimization: Cause or consequence of school maladjustment? », Child Development, vol. 67, n 4, p. 1305-1317.

Kowalski, R.M. et S.P. Limber (2007). «Electronic bullying among middle school students », Journal of Adolescent Health, vol. 41, n 6, p. S22-S30.

Kowalski, R.M., S.P. Limber et P.W. Agatston (2008). Cyber Bullying: Bullying in the Digital Age, Malden (MA), Blackwell Publishing.

Kowalski, R.M., G.W. Giumetti, A.N. Schroeder et M.R. Lattanner (2014). « Bullying in the Digital Age: A Critical Review and Meta-Analysis of Cyberbullying Research Among Youth », Psychological Bulletin, vol. 140, $\mathrm{n}^{\circ}$ 4, p. 1073-1137.

Kumpalainen, K., E. Räsänen et I. Henttoten (1999). « Children involved in bullying: Psychological disturbance and the persistence of the involvement », Child Abuse and Neglect, vol. 23, $\mathrm{n}^{\circ} 12$, p. $1253-1262$. 
Ladd, G.W. (2005). Children's Peer Relations and Social Competence: A Century of Progress, New Haven (CT), Yale University Press.

Le Deuff, O., O. Ertzscheid, D. Boyd, A. Gunther, D. Prud'homme, P. d'Huy, A. Cordier et D. Frochot (2010). «Une évolution des comportements», Documentaliste. Sciences de l'Information, vol. 47, n 1, p. 42-55.

Li, Q. (2006). «Cyberbullying in Schools: A Research of Gender Differences », School Psychology International, vol. 27, $\mathrm{n}^{\circ}$ 2, p. 157-170.

Li, Q. (2007). « New bottle but old wine. A research on cyberbullying in schools », Computers and Human Behavior, vol. 23, n 4, p. 1777-1791.

Li, Q. (2010). « Cyberbullying in High Schools: A Study of Students' Behaviors and Beliefs about This New Phenomenon », Journal of Aggression, Maltreatment \& Trauma, vol. 19, n 4, p. 372392.

Ling, R., et al. (2003). « Mobile communications and social capital in Europe », in K. Nyiri (dir.), Mobile Democracy: Essays on Society, Self and Politics, Vienne, Passagen.

Livingstone, S. (2008). «Taking risky opportunities in youthful content creation: teenagers' use of social networking sites for intimacy, privacy and self-expression », New Media \& Society, vol. 10, $n^{\circ} 3$, p. 393-411.

Ma, X. (2001). « Bullying and Being Bullied: To What Extent Are Bullies Also Victims? », American Educational Research Journal, vol. 38, $\mathrm{n}^{\circ}$ 2, p. 351-370.

Mason, K.L. (2008). « Cyberbullying: A preliminary assessment for the school personnel », Psychology in the Schools, vol. 45, $\mathrm{n}^{\circ}$ 4, p. 323-348.

McGuinness, T.M. (2007). « Dispelling the Myths of Bullying », Journal of Psychological Nursing, vol. $45, n^{\circ} 10$, p. $19-22$.

Menesini, E., M. Modena et F. Tani (2009). «Bullying and Victimization in Adolescence: Concurrent and Stable Roles and Psychological Health Symptoms », Journal of Genetic Psychology, vol. 170, n² 2, p. 115-133.

Mertens, DM. (2010). Research and evaluation in education and psychology: Integrating diversity with quantitative, qualitative, and mixed methods, ( $3^{e}$ éd.), Thousand Oaks (CA), Sage.

Mishna, F., M. Saini et S. Solomon (2009). «Ongoing and online: children and youth's perceptions of cyber bullying », Child Youth Service Review, vol. 31, n 12, p. 1222-1228.

Moffitt, T.E. (1993). «Adolescence-limited and life-course-persistent antisocial behaviour: A developmental taxonomy », Psychological Review, vol. 100, nº 4, p. 674-701.

Mynard, H., et S. Joseph (2000). « Development of the multidimensional peer-victimization scale », Aggressive Behavior, vol. 26, n² 2, p. 169-178. 
Nansel, T.R., M. Overpeck, R.S. Pilla, W.J. Ruan, B. Simons-Morton et P. Scheidt (2001). «Bullying behaviors among US youth: prevalence and association with psychosocal adjustment », Journal of the American Medical Association, vol. 285, n 16, p. 2094-2100.

Nishina, A., J. Juvonen et M.R. Witkow (2005). « Sticks and Stones May Break My Bones, but Names Will Make Me Feel Sick: The Psychosocial, Somatic, and Scholastic Consequences of Peer Harassment », Journal of Clinical Child and Adolescent Psychology, vol. 34, $\mathrm{n}^{\circ}$ 1, p. 3748.

Office des personnes handicapées du Québec (2014) : https://www.ophq.gouv.qc.ca/fileadmin/documents/trousse_Vfinal.pdf.

Ogilvie, D.M. (1987). « The undesired self: A neglected variable in personality research », Journal of Personality and Social Psychology, vol. 52, $n^{\circ} 2$, p. 379-385.

Oksman, V., et P. Rautiainen (2002). « "Perhaps it is a body part": how the mobile phone became an organic part of the everyday lives of Finnish children and teenagers », in J.E. Katz (dir.), Machines that Become Us: The Social Context of Personal Communication Technology, New Brunswick (NJ), Transaction Publishers.

Patchin, J.W., et S. Hinduja (2012). « Cyberbullying: An update and synthesis of the research », in J.W. Patchin et S. Hinduja (dir.). Cyberbullying Prevention and Responses: Expert Perspectives, New York, Routledge, p. 13-35.

Patchin, J.W., et S. Hinduja (2010). « Cyberbullying and self-esteem », Journal of School Health, vol. $80, n^{\circ} 12$, p. $614-621$.

Payne, A.A., D.C. Gottfredson et G.D. Gottfredson (2003). « Schools as communities: the relationships among communal school organizations, student bonding, and school disorder », Criminology, vol. 42, $\mathrm{n}^{\circ} 3$, p. 749-777.

Pereira, C.P. (2015). « Technological culture and new practices of knowledge production »,in E. Théodoropoulou E. (dir.), Philosophie de l'éducation. Aspect de praxis, Athènes, Pedio, p. 219237.

Perren, S., J. Dooley, T. Shaw et D. Cross (2010) «Bullying in school and cyberspace: Associations with depressive symptoms in Swiss and Australian adolescents », Child and Adolescent Psychiatry and Mental Health, vol. 4, $\mathrm{n}^{\circ} 28$.

Perren, S., A. von Wyl, S. Stadelmann, D. Bürgin et K. von Klizing (2006) Associations between behavioral/emotional difficulties in kindergarten children and the quality of their peer relationships. Journal of the American Academy of Child and Adolescent Psychiatry, vol. 45, $\mathrm{n}^{\circ} 7$, p. 867-876.

Puelles, A.A., et P.C. Pereira (2009). « La nueva ciudad de dios: para une acqueologia de la ciudad digital », in P.C. Pereira et J. Bustamante (dir.). A Filosofia e a Cidade, Porto, Edições, Afrontamento.

Raskauskas, J., et A.D. Stoltz (2007). « Involvement in traditional and electronic bullying among adolescents », Developmental Psychology, vol. 43, n³, p. 564-575. 
Reader Goodman, M., E.A. Stormshak et T.J. Dishion (2001). « The significance of peer victimization at two points in development », Journal of Applied developmental Psychology, vol. 22, $n^{\circ} 5$, p. 507-526.

Rincon-Robichaud, M. G.(2003). L'enfant souffre-douleur. L'intimidation à l'école. Montréal, Éditions de l'Homme.

Rigby, K., et P.T. Slee (1991). « Bullying among Australian School Children: Reported Behavior and Attitudes toward Victims », Journal of Social Psychology, vol. 131, n 5, p. 615-627.

Roberts, D.F., et U.L. Foehr (2003). Kids and Media in America, Cambridge (MA), Cambridge University Press.

Salmivalli, C., A. Kaukiainen, L. Kaistaniemi et K.M.J. Lagerspetz (1999). « Self-Evaluated SelfEsteem, Peer-Evaluated Self-Esteem, and Defensive Egotism as Predictors of Adolescents' Participation in Bullying Situations », Personality and Social Psychology Bulletin, vol. 25, $\mathrm{n}^{\circ}$ 10, p. $1268-1278$.

Schneider, S.K., L. O'Donnell, A. Stueve et R.W.S. Coulter (2012). « Cyberbullying, School Bullying, and Psychological Distress: A Regional Census of High School Students », American Journal of Public Health, vol. 102, n¹, p. 171-177.

Schuster, B. (1996). "Rejection, exclusion, and harassment at work and in schools: An integration of results from research on mobbing, bullying and peer rejection », European Psychologist, vol. 1, n 4, p. 293-317.

Seals, D., et J. Young (2003). « Bullying and victimization: prevalence and relationship to gender, grade level, ethnicity, self-esteem and depression », Adolescence, vol. 38, nº 152, p. 735-747.

Sharp, E.H., L.L. Caldwell, J.W. Graham et T.A. Ridenour (2006). « Individual motivation and parental influence on adolescents' experiences of interest in free time: A longitudinal examination », Journal of Youth and Adolescence, vol. 35, n³, p. 359-372.

Silvia, P.J., et K.M. Eddington (2012). « Self and Emotion », in M.R. Leary et J.P. Tangney (dir.), Handbook of Self and Identity ( $2^{\mathrm{e}}$ éd.), New York, Guilford, p. 425-445.

Slonje, R., et P.K. Smith (2008). « Cyberbullying : Another main type of Bullying? », Scandinavian Journal of Psychology, vol. 49, n² 2, p. 147-154.

Smith, P.K., J. Mahdavi, M. Carvalho, S. Fisher, S. Russel et N. Tippet (2008). « Cyberbullying: its nature and impact in secondary school pupils », Journal of Child Psychology and Psychiatry, vol. 49, n 4, p. 376-385.

Spriggs, A.L., R.J. Iannotti, T.R. Nansel et D.L. Haynie (2007). « Adolescent Bullying Involvement and Perceived Family, Peer and School Relations: Commonalitities and Differences Across Race/Ethnicity », Journal of Adolescent Health, vol. 41, nº 3, p. 283-293.

Sticca, F., et S. Perren (2013). « Is Cyberbullying Worse than Traditional Bullying? Examining the Differential Roles of Medium, Publicity, and Anonymity for the Perceived Severity of Bullying », Journal of Youth and Adolescence, vol. 42, $n^{\circ} 5$, p. 739-750. 
Stiegler, B (2008). Prendre soin, tome 1: De la jeunesse et des générations, Paris, Flammarion.

Stover, D. (2005). « Climate and Culture: Why your board should pay attention to the attitudes of students and staff », American School Board Journal, vol. 192, n 12, p. 30-33.

Storch, E.A., M.K. Nock, C. Masia-Warner et M.E. Barlas (2003). « Peer Victimizationand SocialPsychological Adjustment in Hispanic and African-American Children », Journal of Child and Family Studies, vol. 12, no 4, p. 439-452.

Strom, P.S., et R.D. Strom (2005). « Cyberbullying by Adolescents: A Preliminary Assessment », The Educational Forum, vol. 70, $\mathrm{n}^{\circ}$ 1, p. 21-32.

Sullivan, K. (2000). The Anti-Bullying Handbook, New York, Oxford University Press.

Tassoni, P. (2007). Child Development: 6 to 16 Years, Oxford, Heinemann.

Tokunaga, R.S. (2010). « Following you home from school : a critical review and synthesis of research on cyberbullying victimization », Computer in Human Behavior, vol. 26, $\mathrm{n}^{\circ} 3$, p. 277287.

Trachtenbroit, M.L.B. (2012). Cyberbullying, school violence, and youth suicide, thèse soutenue à University of Southern Mississipi, Hattiesburg (MS), Dissertation Abstracts International Section A: Humanities and Social Sciences, 73(4-A), 1255.

Ttofi, M.M., et D.P. Farrington (2009). « What works in preventing bullying: Effective, elements of anti-bullying programmes », Journal of Aggression, Conflict and Peace. Research, vol. 1, n 1, p. 13-24.

Turkle, S. (1995). Life on the Screen: Identity in the Age of the Internet, New York, Simon and Schuster.

Turkle, S. (1997). «Multiple subjectivity and virtual community at the end of the Freudian century », Sociological Inquiry, vol. $67, \mathrm{n}^{\circ} 1, \mathrm{p} .72-84$.

Turkle, S. (2004). "How computers change the way we think », The Chronicle of Higher Education, vol. 50, $\mathrm{n}^{\circ}$ 21, p. B26.

Twyman, K., C. Saylor, L.A. Taylor et C. Comeaux (2010). « Comparing Children and Adolescents Engaged in Cyberbullying to Matched Peers », Cyberpsychology, Behavior, and Social Networking, vol. 13, $\mathrm{n}^{\circ}$ 2, p. 195-199.

Varjas, K., C.C. Henrich et J. Meyers (2009). « Urban Middle School Students' Perceptions of Bullying, Cyberbullying, and School Safety », Journal of School Violence, vol. 8, $\mathrm{n}^{\circ}$ 2, p. 159176.

Wang, J., R.J. lannotti et T.R. Nasel (2009). « School Bullying among Adolescents in the United States: Physical, Verbal, Relational, and Cyber », Journal of Adolescent Health, vol. 45, $n^{\circ} 4$, p. 368-375. 
Wild, L.G., A.J. Flisher, A. Bhana et C. Lombard (2004). « Associations among adolescent risk behaviours and self-esteem in six domains », Journal of Child Psychology and Psychiatry, vol. $45, n^{\circ} 8$, p. 1454-1467.

Willard, N.E. (2007a). «The Authority and Responsibility of School Officials In Responding to Cyberbullying », Journal of Adolescent Health, vol. 41, n 6, p. S64-S65.

Willard, N.E. (2007b). Cyberbullying and Cyberthreats: Responding to the Challenge of Online Social Agression, Threats, and Distress, Champaign (IL), Research Press.

Williams, K.R., et N.G. Guerra (2007). «Prevalence and Predictors on Internet Bullying », Journal of Adolescent Health, vol. 41, n 6, p. S14-S21.

Wolak, J., K.J. Mitchell et D. Finkelhor (2007). « Does Online Harassment Constitute Bullying? An Exploration of Online Harassment by Known Peers and Online-Only Contacts », Journal of Adolescent Health, vol. 41, n 6, p. S51-S58.

Yang, Y., J. Yen, C. Ko, C. Cheng et C. Yen (2010). « The association between problematic cellular phone use and risky behaviors and low self-esteem among.

Taiwanese adolescents ». BMC Public Health, vol. 10, $\mathrm{n}^{\circ}$ 217, p. 1-8. Ybarra, M.L. (2004). «Linkages between depressive symptomatology and Internet harassment among young regular Internet users », Cyberpsychology \& Behavior, vol. 7, n² 2, p. 247-257.

Ybarra, M.L., K.J. Mitchell, J. Wolak et D. Finkelhor (2006). « Examining Characteristics and Associated Distress Related to Internet Harassment: Findings From the Second Youth Internet Safety Survey », Pediatrics, vol. 118, nº 4, p. e1169-e1177.

Ybarra, M.L., M. Diener-West et P.J. Leaf (2007). «Examining the Overlap in Internet Harassment and School Bullying: Implications for School Intervention », Journal of Adolescent Health, vol. 41, $n^{\circ} 6$, p. S42-S50. 